\title{
Dimensionamento da amostra para a estimação da média de precipitação pluvial mensal em diferentes locais do Estado do Rio Grande do Sul
}

\author{
Sample size dimensioning for estimating the average monthly rainfall in different locations \\ of Rio Grande do Sul State, Brazil
}

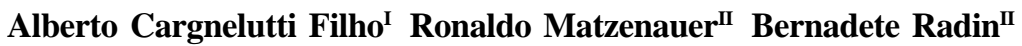 \\ Jaime Ricardo Tavares Maluf ${ }^{\text {II }}$ Lisiane Hauser ${ }^{\text {III }}$
}

RESUMO

Com o objetivo de determinar o tamanho de amostra para a estimação da média de precipitação pluvial mensal de 19 locais do Estado do Rio Grande do Sul, utilizaram-se os dados de precipitação pluvial do período de 1953 a 2007. Em cada uma das 228 séries temporais (12 meses x 19 locais), calcularam-se medidas de tendência central, de variabilidade e de assimetria, e testaram-se a aleatoriedade e a normalidade dos dados. Em seguida, verificou-se a homogeneidade de variâncias entre os meses em cada local $e$ entre os locais em cada mês. Depois, calculou-se o tamanho de amostra em cada mês e local. O tamanho de amostra (número de anos) para a estimação da média de precipitação pluvial mensal é dependente do mês e do local. Para os meses e locais estudados, 51 anos de observações são suficientes para estimar a média de precipitação pluvial mensal, para um erro de estimação igual a $25 \%$ da média estimada, com grau de confiança de $95 \%$.

Palavras-chave: série temporal, erro de estimação, tamanho de amostra, amostragem.

\section{ABSTRACT}

It was used data from 19 locations of the Rio Grande do Sul State, Brazil, collected from 1953 to 2007, with the objective to determine the sample size to estimate the average monthly rainfall. The central tendency, variability and asymmetry was calculated for each of the 228 time series (12 months $x 19$ locations) and the aleatory and normality data were tested. Then it was verified the homogeneity of variance among months in each locality and among places in each month and it was determined the sample size to estimate the average monthly rainfall in each month and locality. The sample size (number of years) to estimate the average monthly rainfall is dependent on the month and locality. One concluded that 51 years of data are enough to predict the average monthly rainfall, with an estimation error equal to $25 \%$ of estimated average, with a degree confidence of $95 \%$.

Key words: time series, error of estimation, sample size, sampling.

\section{INTRODUÇÃO}

A precipitação pluvial é importante para o planejamento adequado de atividades agropecuárias e esse elemento meteorológico, em um determinado período do ano e local, deve ser estimado com a uma determinada precisão estipulada pelo pesquisador. Nesse sentido, séries temporais de 30 anos de dados climáticos (normais climatológicas) têm sido utilizadas para estimar os parâmetros de elementos meteorológicos, dentre eles, a média de precipitação pluvial mensal. É evidente que a estimativa da média obtida a partir de amostra de 30 anos de observações está associada a um erro, devido à variabilidade das observações entre os anos.

A variabilidade dos dados climáticos entre as épocas do ano, os locais e os elementos meteorológicos tem sido contemplada em estudos de CARGNELUTTIFILHO et al. (2006a, 2006b, 2007, 2009), no Estado do Rio Grande do Sul. Os autores evidenciaram que, para uma mesma precisão, há variabilidade do tamanho de amostra (número de anos)

IDepartamento de Fitotecnia, Centro de Ciências Rurais (CCR), Universidade Federal de Santa Maria (UFSM), 97105-900, Santa Maria, RS, Brasil. E-mail: cargnelutti@pq.cnpq.br. Autor para correspondência.

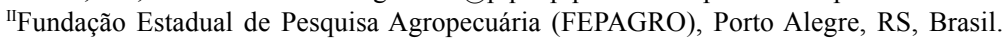

IIICurso de Estatística, Departamento de Estatística, Universidade Federal do Rio Grande do Sul (UFRGS), Porto Alegre, RS, Brasil. 
no tempo (meses e decêndios) e no espaço (locais) para obtenção de estimativas de temperatura mínima (CARGNELUTTI FILHO et al., 2006a) e máxima do ar média mensal (CARGNELUTTI FILHO et al., 2006b) e de radiação solar global média decendial (CARGNELUTTI FILHO et al., 2007). Esses autores concluíram ainda que maior tamanho de amostra para a estimativa de temperatura mínima é necessário nos meses de maio, junho e julho e para a estimativa de temperatura máxima nos meses de maio, junho, julho e agosto. Em relação à radiação solar global média decendial, concluíram que maior tamanho de amostra é necessário nos decêndios dos meses de junho, julho, agosto e setembro em relação aos outros meses. Para o Estado de São Paulo, MARTIN et al. (2008) concluíram que há variabilidade temporal e espacial do tamanho de amostra para as estimativas de insolação, de radiação solar global e de radiação fotossinteticamente ativa. Porém, estudos de tamanho de amostra, relacionados à estimativa da média de precipitação pluvial mensal no Estado do Rio Grande do Sul, não foram encontrados.

Ao calcular o tamanho de amostra em um determinado período do ano e local de um determinado elemento meteorológico, é preciso estabelecer um erro de estimação máximo aceitável com um determinado grau de confiança. Em relação à estimativa da média de precipitação pluvial mensal, não há um valor que possa ser definido como erro máximo tolerável. Porém, deve ser considerada a possibilidade de obter uma estimativa da média com a máxima confiabilidade possível. Assim, neste estudo, fixou-se o erro tolerável na estimativa da média (m) de precipitação pluvial mensal de 10 e $25 \%$ da média estimada, ou seja, $0,10 \mathrm{~m}$ e de $0,25 \mathrm{~m}$, com grau de confiança $(1-\alpha)$ de $95 \%$. É evidente que o tamanho de amostra aumenta com a diminuição do erro de estimação assumido, o acréscimo do grau de confiança e o aumento da variabilidade dos dados.

O objetivo deste trabalho foi determinar o tamanho de amostra para a estimação da média de precipitação pluvial mensal em diferentes locais do Estado do Rio Grande do Sul.

\section{MATERIAL E MÉTODOS}

Os dados de precipitação pluvial de 19 estações (locais) agrometeorológicas localizadas no Estado do Rio Grande do Sul foram obtidos junto ao Banco de Dados do Centro de Meteorologia Aplicada, da Fundação Estadual de Pesquisa Agropecuária FEPAGRO/SCT-RS. Eles foram coletados no período de 1953 a 2007 (Tabela 1). Em cada local e ano, somaramse os dados diários de precipitação pluvial, obtendo- se a precipitação pluvial mensal, em $\mathrm{mm} \mathrm{mes}^{-1}$, de cada um dos 12 meses do ano. Assim, formaram-se 228 séries temporais (12 meses x 19 locais), com número diferenciado de anos de observações em cada série, definidas em função da disponibilidade de dados meteorológicos.

Em cada uma das 228 séries temporais de precipitação pluvial mensal, foram calculadas a média (m), a mediana, a variância $\left(\mathrm{s}^{2}\right)$, o desvio padrão (s) e o coeficiente de assimetria. A fim de identificar possíveis tendências de acréscimo ou decréscimo da precipitação pluvial mensal no período estudado, verificou-se a aleatoriedade dos dados em cada série temporal, por meio do teste de sequência (run test) (SPIEGEL et al., 2004; SIEGEL \& CASTELLAN JÚNIOR, 2006). Foi aplicado um teste bilateral a hipótese $\mathrm{H}_{0}$ : a série é aleatória (sem tendência) versus a hipótese $\mathrm{H}_{1}$ : a série não é aleatória (com tendência). No teste, os dados de precipitação pluvial mensal foram utilizados em ordem cronológica, e o número de sequências foi calculado com base em valores menores e maiores que a mediana. A seguir, foi verificada a normalidade dos dados de cada série temporal, por meio do teste de KolmogorovSmirnov (CAMPOS, 1983; SIEGEL \& CASTELLAN JÚNIOR, 2006).

Aplicou-se o teste de Bartlett (STEEL et al., 1997) aos dados de precipitação pluvial mensal, para verificar a homogeneidade de variâncias entre os meses do ano (12 variâncias - variabilidade temporal), em cada local (19 testes), e entre os locais (19 variâncias variabilidade espacial), em cada um dos 12 meses do ano (12 testes).

Calculou-se o tamanho de amostra $(\eta)$ para a estimação da média de precipitação pluvial mensal de cada uma das 228 séries temporais. Nesses cálculos, consideraram-se semiamplitudes do intervalo de confiança (erro de estimação) iguais a 10 e $25 \%$ da estimativa da média (m) de precipitação pluvial mensal, em mm mes ${ }^{-1}$, com grau de confiança $(1-\alpha)$ de $95 \%$.

Usou-se a expressão $\left.\eta=\frac{\mathrm{t}_{\alpha / 2}^{2} \mathrm{~s}^{2}}{(\text { erro de estimação) }}\right)^{2} \quad$ FONSECA

\& MARTINS 1995; BARBETTA et al., 2004; BUSSAB \& MORETTIN, 2004; SPIEGEL et al., 2004), na qual o erro de estimação é a semiamplitude do intervalo de confiança (fixado em $10 \%$ e $25 \% \mathrm{de} \mathrm{m}$ ); $\mathrm{t}_{\alpha / 2}$ é o valor da distribuição t de Student, cuja área à direita é igual a $\alpha / 2$, isto é, é o valor de $\mathrm{t}$, tal que: $\mathrm{P}\left(\mathrm{t}>\mathrm{t}_{\alpha / 2}\right)=\alpha / 2, \operatorname{com}(\mathrm{n}-1)$ graus de liberdade, $\operatorname{com} \alpha=5 \%$ de probabilidade de erro; n é o número de anos de observações em cada série; e s ${ }^{2}$ é a estimativa de variância. $\mathrm{O}$ tamanho de amostra $(\eta)$ foi calculado iterativamente até sua convergência. 
Dimensionamento da amostra para a estimação da média de precipitação pluvial mensal em diferentes locais...

Tabela 1 - Altitude, latitude e longitude, período de observação da precipitação pluvial mensal e valor calculado da estatística do teste de Bartlett $\left(\chi_{\text {calc }}^{2}\right)$ das variâncias entre os meses em cada local e das variâncias entre os locais em cada mês.

\begin{tabular}{|c|c|c|c|c|c|c|c|}
\hline Local & Altitude (m) & Latitude (S) & Longitude (W) & Período & $\chi_{\text {calc }}^{2}$ & Mês & $\chi_{\text {calc }}^{2}$ \\
\hline Cachoeirinha & 4 & $29^{\circ} 57^{\prime} 36^{\prime \prime}$ & $51^{\circ} 04^{\prime} 22^{\prime \prime}$ & $1975-2007$ & $19,76^{*}$ & Janeiro & $64,62 *$ \\
\hline Caxias do Sul & 787 & $29^{\circ} 10^{\prime} 25^{\prime}$, & $51^{\circ} 12^{\prime} 21^{\prime \prime}$ & $1986-2007$ & $19,80 *$ & Fevereiro & $41,74 *$ \\
\hline Cruz Alta & 473 & $28^{\circ} 38^{\prime} 21^{\prime \prime}$ & $53^{\circ} 36^{\prime} 34^{\prime \prime}$ & $1973-2007$ & $19,74^{*}$ & Março & $60,25 *$ \\
\hline Encruzilhada do Sul & 420 & $30^{\circ} 32^{\prime} 35^{\prime}$, & $52^{\circ} 31^{\prime} 20^{\prime \prime}$ & $1958-2007$ & $10,04^{\mathrm{ns}}$ & Abril & $85,30 *$ \\
\hline Erechim & 760 & $27^{\circ} 37^{\prime} 46^{\prime \prime}$ & $52^{\circ} 16^{\prime} 33^{\prime \prime}$ & $1966-2000$ & $22,92 *$ & Maio & $66,28 *$ \\
\hline Ijuí & 448 & $28^{\circ} 23^{\prime} 17^{\prime}$, & $53^{\circ} 54^{\prime} 50^{\prime \prime}$ & $1963-2007$ & $28,92 *$ & Junho & $29,59 *$ \\
\hline Júlio de Castilhos & 514 & $29^{\circ} 13^{\prime} 26^{\prime \prime}$ & $53^{\circ} 40^{\prime} 45^{\prime \prime}$ & $1956-2007$ & $10,52^{\mathrm{ns}}$ & Julho & $83,93 *$ \\
\hline Maquiné & 32 & $29^{\circ} 40^{\prime} 49^{\prime \prime}$ & $50^{\circ} 13^{\prime} 56^{\prime \prime}$ & $1956-2007$ & $36,78^{*}$ & Agosto & $41,70 *$ \\
\hline Quaraí & 100 & $30^{\circ} 23^{\prime} 17^{\prime}$, & $56^{\circ} 26^{\prime} 53^{\prime \prime}$ & $1966-2007$ & $79,99 *$ & Setembro & $42,16^{*}$ \\
\hline Rio Grande & 15 & $32^{\circ} 01^{\prime} 44^{\prime \prime}$ & $52^{\circ} 15^{\prime} 37^{\prime \prime}$ & $1953-2007$ & $45,40 *$ & Outubro & $58,83^{*}$ \\
\hline Santa Maria & 95 & $29^{\circ} 41^{\prime} 25^{\prime}$, & $53^{\circ} 48^{\prime} 42^{\prime \prime}$ & $1963-2007$ & $10,74^{\mathrm{ns}}$ & Novembro & $45,83 *$ \\
\hline Santa Rosa & 273 & $27^{\circ} 51^{\prime} 50^{\prime \prime}$ & $54^{\circ} 29^{\prime} 03^{\prime \prime}$ & $1975-2007$ & $26,75^{*}$ & Dezembro & $53,14 *$ \\
\hline Santana do Livramento & 210 & $30^{\circ} 53^{\prime} 18^{\prime \prime}$ & $55^{\circ} 31^{\prime} 56^{\prime \prime}$ & $1966-2007$ & $12,17^{\mathrm{ns}}$ & & \\
\hline São Borja & 99 & $28^{\circ} 39^{\prime} 44^{\prime \prime}$ & $56^{\circ} 00^{\prime} 15^{\prime}$, & $1956-2007$ & $53,29 *$ & & \\
\hline São Gabriel & 109 & $30^{\circ} 27^{\prime} 27^{\prime \prime}$ & $54^{\circ} 19^{\prime} 01^{\prime \prime}$ & $1963-2007$ & $15,42^{\mathrm{ns}}$ & & \\
\hline Taquari & 76 & $29^{\circ} 48^{\prime} 15^{\prime \prime}$ & $51^{\circ} 49^{\prime} 30^{\prime \prime}$ & $1963-2007$ & $16,74^{\mathrm{ns}}$ & & \\
\hline Uruguaiana & 74 & $29^{\circ} 45^{\prime} 23^{\prime \prime}$ & $57^{\circ} 05^{\prime} 12^{\prime}$, & $1963-2007$ & $73,63^{*}$ & & \\
\hline Vacaria & 955 & $28^{\circ} 30^{\prime} 09^{\prime}$, & $50^{\circ} 56^{\prime} 12^{\prime \prime}$ & $1966-2007$ & $15,35^{\mathrm{ns}}$ & & \\
\hline Veranópolis & 705 & $28^{\circ} 56^{\prime} 14^{\prime \prime}$ & $51^{\circ} 33^{\prime} 11^{\prime \prime}$ & $1956-2007$ & $29,50 *$ & & \\
\hline
\end{tabular}

* Significativo a $5 \%$ de probabilidade de erro pelo teste de Bartlett. ${ }^{\text {ns }}=$ Não-significativo.

\section{RESULTADOS E DISCUSSÃO}

A mediana da precipitação pluvial mensal oscilou entre 56mm no mês de dezembro, em Rio Grande, e $199 \mathrm{~mm}$ no mês de outubro, em Santa Rosa. Isso significa que, em $50 \%$ dos anos, a precipitação pluvial mensal no mês de dezembro, em Rio Grande, é inferior a $56 \mathrm{~mm}$ e em $50 \%$ dos anos é superior a esse valor. Já em Santa Rosa, no mês de outubro, a precipitação pluvial mensal em 50\% dos anos é inferior a 199mm e em 50\% dos anos é superior (Tabela 2).

O coeficiente de assimetria da precipitação pluvial mensal oscilou entre $-0,56$ no mês de abril, em Caxias do Sul, e 2,68 no mês de junho, em São Borja. Valores abaixo e acima de zero indicam, respectivamente, assimetria negativa e positiva da distribuição dos dados. Em 223 séries temporais $(97,8 \%)$, o coeficiente de assimetria foi maior que zero, indicando assimetria positiva da precipitação pluvial mensal. Neste estudo, a média das estimativas da média (m) de precipitação pluvial mensal das 228 séries temporais foi de $130 \mathrm{~mm} \mathrm{mês}^{-1}$ (Tabela 3 ), e a média das 228 medianas foi de $118 \mathrm{~mm} \mathrm{mês}^{-1}$ (Tabela 2). Ainda, em 209 séries temporais $(91,7 \%)$, a mediana foi inferior à média (Tabelas 2 e 3). Esses resultados estão de acordo com a afirmação de que, em distribuições assimétricas positivas, a mediana é inferior à média (FONSECA \& MARTINS 1995; BARBETTA et al., 2004). Em termos práticos, isso significa afirmar que, em mais de $50 \%$ dos anos, de uma determinada série histórica, a precipitação pluvial mensal é inferior à média.

O desvio padrão oscilou entre $45 \mathrm{~mm}$ no mês de março, em Cachoeirinha, e no mês de agosto, em Uruguaiana, e $133 \mathrm{~mm}$ no mês de abril, em Quarai, e a média dos 228 desvios padrão foi de $77 \mathrm{~mm} \mathrm{mês}^{-1}$ (Tabela 3). Ocorrência de elevadas quantidades de precipitação pluvial e ausência de precipitação pluvial, em determinadas séries temporais, inflacionam o desvio padrão e a estimativa do tamanho de amostra. Considerando o fato que esses valores ocorrem nas séries temporais, sua manutenção no estudo do dimensionamento de amostra é adequada.

Em 215 séries temporais de precipitação pluvial mensal $(94,3 \%)$, o teste de aleatoriedade revelou distribuição aleatória dos dados $(\mathrm{P}>0,05)$ (Tabela 4). Isso caracteriza, de maneira geral, séries temporais não tendenciosas, ou seja, não houve tendência de acréscimo ou decréscimo da precipitação pluvial mensal no período estudado. SIEGEL \& CASTELLAN JÚNIOR (2006) salientam que, se um pesquisador pretende tirar alguma conclusão sobre uma população usando a informação contida em uma amostra dessa população, a amostra deve ser aleatória. 
Cargnelutti Filho et al.

Tabela 2 - Estimativas da mediana, em mm, e da assimetria da precipitação pluvial mensal em locais do Estado do Rio Grande do Sul.

\begin{tabular}{|c|c|c|c|c|c|c|c|c|c|c|c|c|}
\hline \multirow{2}{*}{ Local } & \multicolumn{12}{|c|}{ 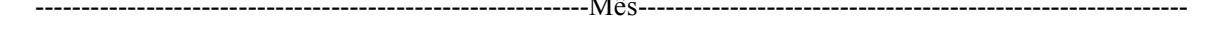 } \\
\hline & Jan & Fev & Mar & Abr & Mai & Jun & Jul & Ago & Set & Out & Nov & Dez \\
\hline & \multicolumn{12}{|c|}{---------------------------------------------------------------Mediana------------------------------------------------------------ } \\
\hline Cachoeirinha & 117 & 93 & 83 & 99 & 84 & 121 & 160 & 113 & 141 & 113 & 108 & 97 \\
\hline Caxias do Sul & 166 & 134 & 122 & 165 & 110 & 149 & 165 & 107 & 146 & 174 & 114 & 113 \\
\hline Cruz Alta & 143 & 126 & 101 & 123 & 113 & 141 & 130 & 129 & 152 & 147 & 124 & 105 \\
\hline Encruzilhada do Sul & 113 & 113 & 112 & 94 & 103 & 135 & 132 & 111 & 140 & 132 & 96 & 95 \\
\hline Erechim & 167 & 156 & 129 & 117 & 121 & 155 & 143 & 144 & 152 & 168 & 136 & 125 \\
\hline Ijuí & 128 & 128 & 99 & 127 & 97 & 122 & 111 & 134 & 155 & 152 & 125 & 136 \\
\hline Júlio de Castilhos & 118 & 106 & 115 & 116 & 97 & 117 & 106 & 118 & 135 & 142 & 112 & 106 \\
\hline Maquiné & 162 & 180 & 167 & 96 & 77 & 97 & 99 & 116 & 148 & 139 & 112 & 131 \\
\hline Quaraí & 105 & 132 & 103 & 131 & 106 & 86 & 84 & 66 & 93 & 137 & 116 & 118 \\
\hline Rio Grande & 101 & 89 & 93 & 69 & 78 & 99 & 122 & 129 & 131 & 98 & 74 & 56 \\
\hline Santa Maria & 118 & 98 & 124 & 131 & 113 & 124 & 121 & 101 & 134 & 139 & 116 & 92 \\
\hline Santa Rosa & 150 & 117 & 108 & 146 & 120 & 133 & 104 & 105 & 150 & 199 & 141 & 111 \\
\hline Santana do Livramento & 145 & 90 & 126 & 85 & 108 & 108 & 93 & 93 & 108 & 124 & 112 & 83 \\
\hline São Borja & 105 & 99 & 145 & 161 & 109 & 95 & 86 & 83 & 135 & 163 & 118 & 130 \\
\hline São Gabriel & 101 & 89 & 94 & 111 & 104 & 115 & 110 & 82 & 133 & 125 & 102 & 71 \\
\hline Taquari & 115 & 113 & 127 & 100 & 89 & 142 & 146 & 121 & 158 & 133 & 115 & 102 \\
\hline Uruguaiana & 105 & 111 & 111 & 152 & 80 & 75 & 61 & 57 & 87 & 115 & 100 & 90 \\
\hline Vacaria & 128 & 132 & 90 & 99 & 102 & 106 & 128 & 113 & 141 & 123 & 115 & 109 \\
\hline Veranópolis & 142 & 123 & 101 & 116 & 98 & 123 & 135 & 142 & 148 & 145 & 117 & 122 \\
\hline & & & & & & 123 & etria--- & & & & & --- \\
\hline $\begin{array}{l}\text { Cachoeirinha } \\
\text { Caxias do Sul }\end{array}$ & $\begin{array}{l}0,50 \\
0,34\end{array}$ & $\begin{array}{l}1,04 \\
0,70\end{array}$ & $\begin{array}{c}0,94 \\
-0,19\end{array}$ & $\begin{array}{c}0,38 \\
-0,56\end{array}$ & $\begin{array}{l}0,40 \\
0,19\end{array}$ & $\begin{array}{l}1,23 \\
0,03\end{array}$ & $\begin{array}{l}0,20 \\
-0,21\end{array}$ & $\begin{array}{l}0,49 \\
0,63\end{array}$ & $\begin{array}{l}0,49 \\
1,56\end{array}$ & $\begin{array}{l}0,89 \\
0,45\end{array}$ & $\begin{array}{l}0,53 \\
0,67\end{array}$ & $\begin{array}{l}1,65 \\
1,22\end{array}$ \\
\hline Cruz Alta & 0,90 & 0,93 & 0,71 & 1,11 & 0,88 & 0,37 & 0,89 & 0,24 & 0,78 & 1,13 & 1,05 & 1,05 \\
\hline Encruzilhada do Sul & 1,11 & 0,91 & 0,39 & 0,97 & 1,50 & 1,46 & 0,29 & 0,78 & 0,45 & 0,83 & 0,62 & 1,21 \\
\hline Erechim & 1,84 & 1,35 & 1,64 & 0,62 & 1,79 & 0,51 & 2,39 & 0,71 & 0,98 & 1,57 & 0,31 & 1,81 \\
\hline Ijuí & 1,00 & 0,45 & 0,63 & 0,63 & 2,36 & 2,07 & 1,26 & 0,54 & 0,65 & 1,46 & 0,73 & 0,92 \\
\hline Júlio de Castilhos & 0,60 & 0,42 & 0,21 & 0,18 & 1,04 & 0,87 & 0,87 & 0,88 & 0,64 & 1,41 & 1,11 & 1,21 \\
\hline Maquiné & 0,51 & 0,38 & 1,32 & 0,91 & 2,40 & 1,81 & 1,25 & 0,91 & 1,42 & 0,30 & 0,55 & 1,18 \\
\hline Quaraí & 1,39 & 0,69 & 1,12 & 1,24 & 0,77 & 1,00 & 0,85 & 0,75 & 0,74 & 0,78 & 1,29 & 1,49 \\
\hline Rio Grande & 1,75 & 2,56 & 0,59 & 1,63 & 0,77 & 0,64 & 2,25 & 0,71 & 0,65 & 0,79 & 1,44 & 1,65 \\
\hline Santa Maria & 1,23 & 0,83 & 0,61 & 0,45 & 1,66 & 0,67 & 0,53 & 0,88 & 0,50 & 1,08 & 0,64 & 1,06 \\
\hline Santa Rosa & 0,10 & 0,52 & 0,40 & 0,72 & 0,95 & 0,30 & 1,63 & 0,92 & 0,31 & 1,16 & 0,58 & 1,02 \\
\hline Santana do Livramento & 1,82 & 0,70 & 0,81 & 0,82 & 1,19 & 1,03 & 1,16 & 1,08 & 0,87 & 0,98 & 0,62 & 1,55 \\
\hline São Borja & 0,55 & 0,52 & 0,88 & 0,78 & 0,50 & 2,68 & 1,55 & 0,98 & 0,10 & 1,45 & 0,75 & 0,34 \\
\hline São Gabriel & 1,30 & 1,60 & 1,15 & 0,93 & 1,08 & 0,45 & 1,08 & 0,50 & 0,42 & 1,47 & 0,55 & 1,42 \\
\hline Taquari & 0,60 & 0,84 & $-0,12$ & 0,41 & 0,51 & 1,31 & 0,33 & 0,57 & 0,21 & 0,81 & 0,49 & 0,89 \\
\hline Uruguaiana & 1,99 & 0,80 & 1,17 & 0,69 & 0,77 & 1,55 & 0,86 & 0,65 & 1,04 & 1,47 & 1,25 & 1,22 \\
\hline Vacaria & $-0,37$ & 0,09 & 1,24 & 0,81 & 0,59 & 0,58 & 1,35 & 0,28 & 0,24 & 1,40 & 1,34 & 0,80 \\
\hline Veranópolis & 0,08 & 1,28 & 0,26 & 0,38 & 0,98 & 0,67 & 0,91 & 0,81 & 1,32 & 0,70 & 0,38 & 1,38 \\
\hline
\end{tabular}

O teste de Kolmogorov-Smirnov revelou que os dados de 226 séries temporais de precipitação pluvial mensal se ajustam à distribuição normal $(\mathrm{P}>0,05)$ (Tabela 4). Então, de maneira geral, pode-se inferir que as séries temporais são aleatórias e, apesar da assimetria positiva observada, não se afastam da normalidade. $\mathrm{O}$ número de anos de observações oscilou entre 22 e 53 anos, e a média entre as 228 séries temporais foi de 42 anos. De acordo com o teorema limite central, mesmo que a população básica seja não normal, a distribuição da média amostral será aproximadamente normal para amostras superiores a 30 observações (FONSECA \& MARTINS 1995; BUSSAB \& MORETTIN, 2004). Então, diante das considerações em relação à aleatoriedade, à normalidade e ao número de observações, pode-se inferir que os dados dessas 228 séries temporais de precipitação pluvial mensal oferecem credibilidade ao estudo do tamanho de amostra (SIEGEL \& CASTELLAN JÚNIOR, 2006). 
Dimensionamento da amostra para a estimação da média de precipitação pluvial mensal em diferentes locais...

Tabela 3 - Estimativas da média e do desvio padrão, em mm, da precipitação pluvial mensal em locais do Estado do Rio Grande do Sul.

\begin{tabular}{|c|c|c|c|c|c|c|c|c|c|c|c|c|}
\hline \multirow{2}{*}{ Local } & \multicolumn{12}{|c|}{ 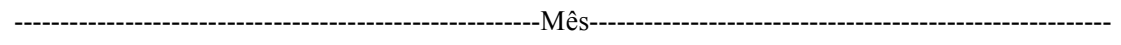 } \\
\hline & Jan & Fev & Mar & Abr & Mai & Jun & Jul & Ago & Set & Out & Nov & Dez \\
\hline Cachoeirinha & 116 & 120 & 90 & 110 & 104 & 141 & 143 & 118 & 134 & 133 & 116 & 107 \\
\hline Caxias do Sul & 167 & 141 & 110 & 150 & 121 & 141 & 160 & 122 & 182 & 172 & 125 & 138 \\
\hline Cruz Alta & 147 & 132 & 122 & 148 & 131 & 142 & 146 & 124 & 164 & 172 & 143 & 134 \\
\hline Encruzilhada do Sul & 119 & 119 & 121 & 108 & 114 & 147 & 135 & 129 & 142 & 134 & 108 & 107 \\
\hline Erechim & 170 & 155 & 131 & 136 & 151 & 171 & 171 & 142 & 172 & 193 & 149 & 150 \\
\hline Ijuí & 136 & 134 & 116 & 137 & 129 & 141 & 124 & 135 & 163 & 180 & 142 & 151 \\
\hline Júlio de Castilhos & 122 & 121 & 120 & 127 & 114 & 138 & 132 & 120 & 147 & 158 & 118 & 127 \\
\hline Maquiné & 175 & 189 & 182 & 104 & 97 & 110 & 113 & 133 & 160 & 144 & 115 & 153 \\
\hline Quaraí & 139 & 155 & 136 & 165 & 129 & 95 & 93 & 72 & 102 & 139 & 134 & 128 \\
\hline Rio Grande & 110 & 111 & 102 & 88 & 85 & 98 & 135 & 132 & 133 & 100 & 88 & 74 \\
\hline Santa Maria & 137 & 116 & 139 & 142 & 114 & 131 & 138 & 117 & 146 & 159 & 119 & 112 \\
\hline Santa Rosa & 145 & 138 & 130 & 157 & 147 & 144 & 117 & 112 & 157 & 210 & 147 & 141 \\
\hline Santana do Livramento & 152 & 128 & 130 & 119 & 113 & 120 & 123 & 103 & 127 & 143 & 110 & 105 \\
\hline São Borja & 133 & 117 & 155 & 180 & 120 & 111 & 88 & 90 & 126 & 168 & 139 & 128 \\
\hline São Gabriel & 111 & 119 & 118 & 130 & 110 & 120 & 126 & 94 & 135 & 133 & 114 & 97 \\
\hline Taquari & 124 & 124 & 116 & 112 & 101 & 145 & 147 & 136 & 153 & 144 & 124 & 105 \\
\hline Uruguaiana & 129 & 140 & 141 & 162 & 109 & 85 & 70 & 69 & 100 & 131 & 109 & 114 \\
\hline Vacaria & 125 & 138 & 107 & 105 & 120 & 115 & 141 & 122 & 148 & 141 & 119 & 123 \\
\hline Veranópolis & 144 & 128 & 114 & 118 & 110 & 132 & 146 & 142 & 172 & 160 & 131 & 149 \\
\hline & & & & & & -Desvi & adrão & & & 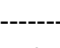 & 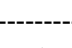 & ----- \\
\hline Cachoeirinha & 66 & 87 & 45 & 56 & 60 & 65 & 70 & 59 & 60 & 72 & 51 & 59 \\
\hline Caxias do Sul & 71 & 74 & 53 & 47 & 64 & 61 & 60 & 76 & 97 & 87 & 55 & 65 \\
\hline Cruz Alta & 82 & 88 & 61 & 99 & 90 & 58 & 86 & 71 & 85 & 99 & 93 & 90 \\
\hline Encruzilhada do Sul & 62 & 76 & 71 & 72 & 79 & 81 & 68 & 77 & 72 & 75 & 64 & 59 \\
\hline Erechim & 100 & 90 & 74 & 87 & 126 & 93 & 127 & 93 & 72 & 106 & 87 & 89 \\
\hline Ijuí & 77 & 87 & 56 & 83 & 116 & 86 & 72 & 85 & 73 & 98 & 90 & 89 \\
\hline Júlio de Castilhos & 63 & 67 & 66 & 69 & 80 & 72 & 73 & 74 & 73 & 89 & 77 & 78 \\
\hline Maquiné & 91 & 97 & 94 & 60 & 78 & 71 & 68 & 83 & 96 & 66 & 58 & 74 \\
\hline Quaraí & 112 & 106 & 95 & 133 & 90 & 57 & 66 & 50 & 61 & 65 & 99 & 88 \\
\hline Rio Grande & 81 & 91 & 68 & 70 & 57 & 55 & 102 & 76 & 73 & 59 & 67 & 56 \\
\hline Santa Maria & 83 & 66 & 84 & 87 & 80 & 67 & 81 & 77 & 83 & 96 & 78 & 67 \\
\hline Santa Rosa & 76 & 86 & 73 & 101 & 102 & 69 & 80 & 76 & 53 & 108 & 84 & 99 \\
\hline Santana do Livramento & 98 & 93 & 72 & 93 & 76 & 69 & 92 & 65 & 77 & 77 & 73 & 87 \\
\hline São Borja & 93 & 72 & 88 & 106 & 86 & 81 & 53 & 58 & 56 & 102 & 86 & 67 \\
\hline São Gabriel & 68 & 93 & 81 & 89 & 74 & 64 & 74 & 60 & 71 & 83 & 73 & 71 \\
\hline Taquari & 66 & 67 & 46 & 72 & 66 & 66 & 71 & 71 & 75 & 80 & 61 & 60 \\
\hline Uruguaiana & 104 & 95 & 95 & 102 & 85 & 53 & 50 & 45 & 61 & 89 & 69 & 75 \\
\hline Vacaria & 54 & 63 & 62 & 67 & 78 & 56 & 89 & 71 & 77 & 75 & 61 & 73 \\
\hline Veranópolis & 61 & 69 & 48 & 68 & 72 & 64 & 77 & 82 & 92 & 78 & 68 & 84 \\
\hline
\end{tabular}

O teste de Bartlett (STEEL et al., 1997) aplicado entre as variâncias dos 12 meses, em cada local, revelou que estas foram heterogêneas $(\mathrm{P} \leq 0,05)$ em 12 locais (63,16\% dos locais), indicando que o tamanho de amostra para a estimativa da média de precipitação pluvial mensal é diferenciado entre os meses (Tabela 1). Nos outros sete locais (Encruzilhada do Sul, Júlio de Castilhos, Santa Maria, Santana do Livramento, São Gabriel, Taquari e Vacaria), as variâncias foram homogêneas, podendo-se inferir que o uso da média do tamanho de amostra entre os meses é adequado para estimar a média de precipitação pluvial mensal nesses locais. Entre as variâncias dos 19 locais, em cada mês, o teste de Bartlett revelou variâncias heterogêneas para os 12 meses do ano, o que revela a necessidade de determinar o tamanho de amostra em cada local (Tabela 1). Variabilidade temporal (entre decêndios e meses) e espacial (entre locais) do tamanho 
Cargnelutti Filho et al.

Tabela 4 - Resultados dos testes de aleatoriedade (Run test) e de normalidade (Kolmogorov-Smirnov) em relação a 228 séries temporais de precipitação pluvial mensal em locais do Estado do Rio Grande do Sul.

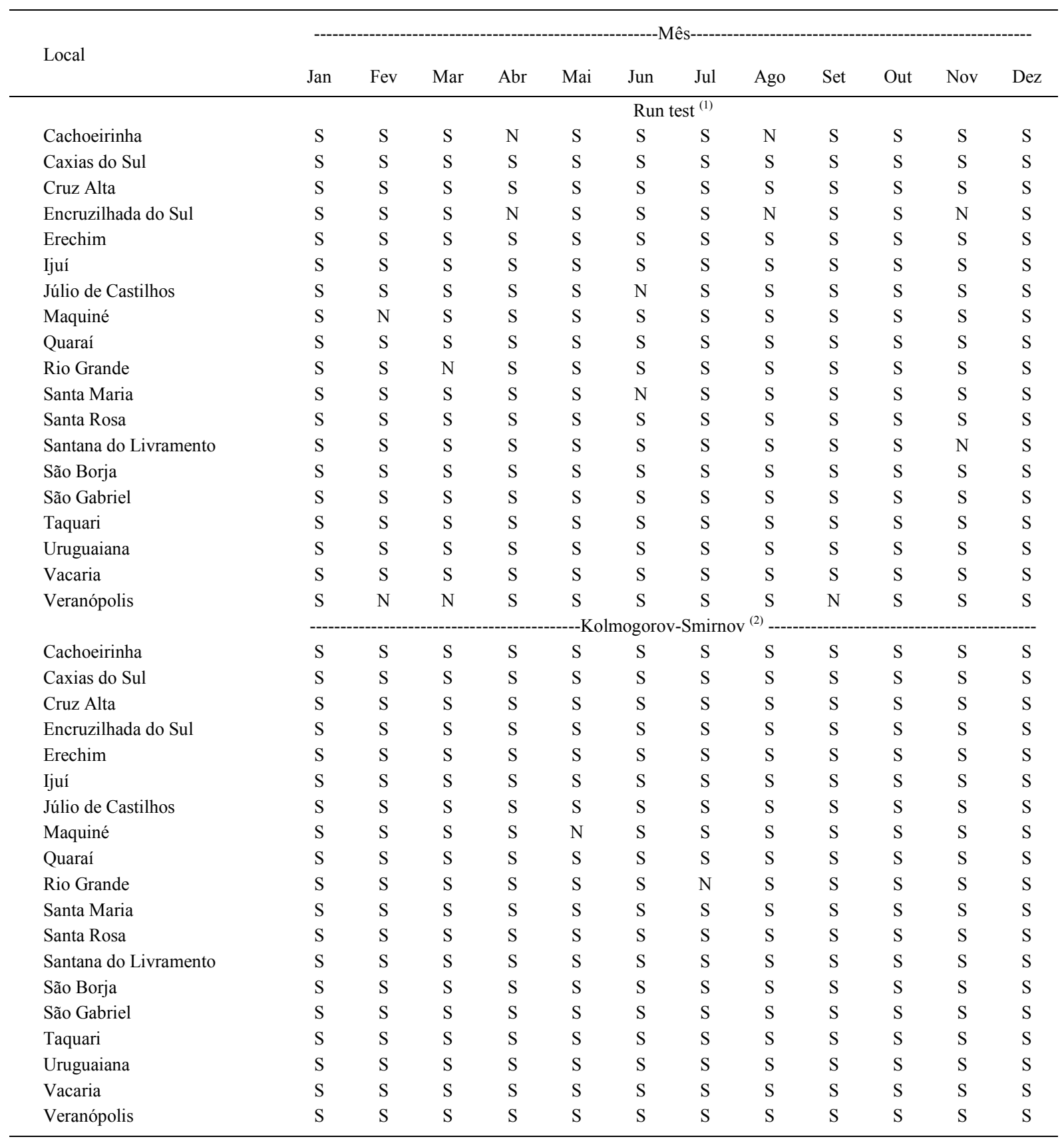

${ }^{(1)} \mathrm{S}=$ Sequência aleatória, pelo Run test $(\mathrm{P}>0,05) . \mathrm{N}=$ Não aleatória $(\mathrm{P} \quad 0,05)$.

${ }^{(2)} \mathrm{S}=$ Distribuição normal, pelo teste Kolmogorov-Smirnov $(\mathrm{P}>0,05) . \mathrm{N}=$ Não nomal $(\mathrm{P} \quad 0,05)$.

de amostra para a estimação de médias de elementos meteorológicos foi constatada no Estado do Rio Grande do Sul (CARGNELUTTI FILHO et al., 2006a, 2006b, 2007) e no Estado de São Paulo (MARTIN et al., 2008).

De maneira geral, os resultados indicam a necessidade de utilizar a maior das 228 variâncias estimadas (228 séries temporais) para determinar um tamanho de amostra único para esses meses e locais, a partir de um erro tolerável e grau de confiança fixo. Por outro lado, a variabilidade existente entre as 228 séries temporais possibilita a identificação de meses e locais com maior e menor tamanho de amostra necessário para estimar a média de precipitação pluvial mensal. 
O tamanho de amostra, para a estimação da média de precipitação pluvial mensal em cada mês e local, com semiamplitude do intervalo de confiança igual a $10 \%$ da média estimada, em mm mes ${ }^{-1}$, e grau de confiança de $95 \%$, oscilou entre 41 anos no mês de abril, em Caxias do Sul, e 301 anos no mês de maio, em Ijuí (Tabela 5). Portanto, em relação ao mês de abril em
Caxias do Sul, pode-se inferir, com $95 \%$ de confiança, que o intervalo de confiança da média de precipitação pluvial mensal obtida com 41 anos de observações é de $\mathrm{m} \pm 0,10 \mathrm{~m}$. Em outro extremo, a precisão de $\mathrm{m} \pm 0,10 \mathrm{~m}$ é obtida com 301 anos de observações, em relação ao mês de maio em Ijuí. Esses resultados confirmam a variabilidade existente entre meses dentro dos locais

Tabela 5 - Tamanho de amostra (número de anos) para a estimativa da média (m) de precipitação pluvial mensal em locais do Estado do Rio Grande do Sul com $95 \%$ de confiança e semiamplitude do intervalo de confiança de $0,10 \mathrm{~m}$ e de $0,25 \mathrm{~m}$.

\begin{tabular}{|c|c|c|c|c|c|c|c|c|c|c|c|c|c|}
\hline Local & Jan & Fev & Mar & Abr & Mai & Jun & Jul & Ago & Set & Out & Nov & Dez & Máximo \\
\hline \multicolumn{14}{|c|}{-----------------------Semiamplitude do intervalo de confiança de 0,10m---------------------- } \\
\hline Cachoeirinha & 125 & 206 & 98 & 104 & 131 & 85 & 94 & 97 & 79 & 115 & 78 & 118 & 206 \\
\hline Caxias do Sul & 72 & 109 & 93 & 41 & 110 & 74 & 58 & 153 & 111 & 100 & 76 & 89 & 153 \\
\hline Cruz Alta & 122 & 176 & 98 & 174 & 185 & 67 & 137 & 128 & 107 & 129 & 163 & 176 & 185 \\
\hline Encruzilhada do Sul & 105 & 157 & 135 & 171 & 188 & 121 & 99 & 141 & 102 & 125 & 139 & 121 & 188 \\
\hline Erechim & 135 & 134 & 125 & 161 & 270 & 116 & 214 & 168 & 71 & 120 & 133 & 139 & 270 \\
\hline Ijuí & 126 & 162 & 92 & 145 & 301 & 146 & 132 & 155 & 80 & 116 & 158 & 136 & 301 \\
\hline Júlio de Castilhos & 105 & 121 & 117 & 115 & 194 & 108 & 122 & 148 & 97 & 124 & 166 & 149 & 194 \\
\hline Maquiné & 106 & 104 & 107 & 130 & 253 & 164 & 144 & 152 & 141 & 83 & 100 & 93 & 253 \\
\hline Quaraí & 249 & 181 & 189 & 252 & 193 & 142 & 193 & 190 & 138 & 88 & 210 & 184 & 252 \\
\hline Rio Grande & 215 & 265 & 176 & 250 & 180 & 125 & 226 & 129 & 117 & 135 & 228 & 220 & 265 \\
\hline Santa Maria & 146 & 128 & 142 & 146 & 192 & 102 & 135 & 169 & 126 & 143 & 167 & 139 & 192 \\
\hline Santa Rosa & 108 & 153 & 122 & 164 & 190 & 92 & 184 & 178 & 46 & 105 & 130 & 190 & 190 \\
\hline Santana do Livramento & 163 & 205 & 122 & 236 & 178 & 129 & 219 & 155 & 144 & 115 & 170 & 264 & 264 \\
\hline São Borja & 194 & 148 & 126 & 137 & 197 & 206 & 145 & 162 & 78 & 145 & 152 & 109 & 206 \\
\hline São Gabriel & 145 & 241 & 183 & 186 & 177 & 113 & 134 & 162 & 107 & 151 & 161 & 210 & 241 \\
\hline Taquari & 111 & 113 & 64 & 160 & 168 & 83 & 92 & 108 & 94 & 123 & 96 & 127 & 168 \\
\hline Uruguaiana & 253 & 179 & 178 & 157 & 232 & 155 & 197 & 169 & 147 & 182 & 154 & 171 & 253 \\
\hline Vacaria & 74 & 83 & 129 & 159 & 166 & 94 & 157 & 132 & 107 & 111 & 104 & 136 & 166 \\
\hline Veranópolis & 73 & 115 & 71 & 130 & 167 & 94 & 108 & 130 & 113 & 94 & 108 & 126 & 167 \\
\hline \multirow[t]{2}{*}{ Máximo } & 253 & 265 & 189 & 252 & 301 & 206 & 226 & 190 & 147 & 182 & 228 & 264 & 301 \\
\hline & \multicolumn{13}{|c|}{---------------------Semiamplitude do intervalo de confiança de $0,25 \mathrm{~m}--------------------$} \\
\hline Cachoeirinha & 23 & 35 & 18 & 19 & 23 & 16 & 18 & 18 & 15 & 21 & 15 & 21 & 35 \\
\hline Caxias do Sul & 14 & 20 & 17 & 9 & 20 & 14 & 12 & 27 & 20 & 19 & 15 & 17 & 27 \\
\hline Cruz Alta & 22 & 31 & 18 & 30 & 32 & 13 & 24 & 23 & 20 & 23 & 29 & 31 & 32 \\
\hline Encruzilhada do Sul & 19 & 28 & 24 & 30 & 33 & 22 & 18 & 25 & 19 & 22 & 25 & 22 & 33 \\
\hline Erechim & 24 & 24 & 22 & 28 & 46 & 21 & 37 & 29 & 14 & 22 & 24 & 25 & 46 \\
\hline Ijuí & 23 & 28 & 17 & 26 & 51 & 26 & 24 & 27 & 15 & 21 & 28 & 24 & 51 \\
\hline Júlio de Castilhos & 19 & 22 & 21 & 21 & 34 & 20 & 22 & 26 & 18 & 22 & 29 & 26 & 34 \\
\hline Maquiné & 19 & 19 & 20 & 23 & 43 & 29 & 25 & 27 & 25 & 16 & 18 & 17 & 43 \\
\hline Quaraí & 42 & 32 & 33 & 43 & 33 & 25 & 33 & 33 & 25 & 17 & 36 & 32 & 43 \\
\hline Rio Grande & 37 & 45 & 31 & 43 & 31 & 22 & 39 & 23 & 21 & 24 & 39 & 38 & 45 \\
\hline Santa Maria & 26 & 23 & 25 & 26 & 33 & 19 & 24 & 29 & 23 & 25 & 29 & 25 & 33 \\
\hline Santa Rosa & 20 & 27 & 22 & 29 & 33 & 17 & 32 & 31 & 10 & 19 & 23 & 33 & 33 \\
\hline Santana do Livramento & 29 & 35 & 22 & 40 & 31 & 23 & 37 & 27 & 26 & 21 & 30 & 45 & 45 \\
\hline São Borja & 33 & 26 & 23 & 24 & 34 & 35 & 26 & 28 & 15 & 26 & 27 & 20 & 35 \\
\hline São Gabriel & 26 & 41 & 32 & 32 & 31 & 21 & 24 & 28 & 20 & 27 & 28 & 36 & 41 \\
\hline Taquari & 20 & 21 & 13 & 28 & 29 & 16 & 17 & 20 & 18 & 22 & 18 & 23 & 29 \\
\hline Uruguaiana & 43 & 31 & 31 & 28 & 40 & 27 & 34 & 29 & 26 & 32 & 27 & 30 & 43 \\
\hline Vacaria & 14 & 16 & 23 & 28 & 29 & 17 & 28 & 24 & 20 & 20 & 19 & 24 & 29 \\
\hline Veranópolis & 14 & 21 & 14 & 23 & 29 & 17 & 20 & 23 & 21 & 18 & 20 & 23 & 29 \\
\hline Máximo & 43 & 45 & 33 & 43 & 51 & 35 & 39 & 33 & 26 & 32 & 39 & 45 & 51 \\
\hline
\end{tabular}


(temporal) e entre locais dentro de meses (espacial). Então, 301 anos de observações forneceriam estimativas de média de precipitação pluvial mensal, com 95\% de confiabilidade de que o erro máximo é $0,10 \mathrm{~m}$, independentemente do mês e local.

Do ponto de vista prático, dispor de séries temporais com 301 anos de observações, para estimar a média de precipitação pluvial mensal, é difícil. Assim, menores tamanhos de amostra foram determinados com base em uma semiamplitude do intervalo de confiança igual a $25 \%$ da estimativa da média $(\mathrm{m})$ de precipitação pluvial mensal (Tabela 5). Não cabe aqui o julgamento do erro de estimação máximo aceitável, ficando essa tarefa ao usuário dessas informações, de acordo com a disponibilidade de dados e a precisão desejada. Portanto, tomando-se como referência o maior tamanho de amostra (mês de maio em Ijuí), pode-se inferir, com $95 \%$ de confiança, que, com o uso de 51 anos de observações, o erro máximo na estimativa da média (m) de precipitação pluvial mensal será de $\pm 25 \%$ de m, independentemente do mês e local.

Esses resultados indicam que o valor de até 51 anos de observações, superior ao utilizado nas normais climatológicas (30 anos), é suficiente para estimar a média de precipitação pluvial mensal, para uma semiamplitude do intervalo de confiança igual a $25 \%$ de $\mathrm{m}$, com grau de confiança de $95 \%$. Portanto, as estimativas de médias de normais climatológicas desses locais, com base em 30 anos de observações, possuem um erro de estimação menor ou maior que $25 \%$, com grau de confiança de $95 \%$. Assim, o uso de 30 anos, de forma generalizada, não contempla a variabilidade dos dados de cada local e época do ano, o que leva a estimativas da média de precipitação pluvial mensal com erros diferenciados.

\section{CONCLUSÕES}

O tamanho de amostra (número de anos), para a estimativa da média de precipitação pluvial mensal no Estado do Rio Grande do Sul, é dependente do mês e do local. Para os meses e locais estudados, 51 anos de observações são suficientes para estimar a média de precipitação pluvial mensal, para um erro de estimação igual a $25 \%$ da média estimada, com grau de confiança de $95 \%$.

\section{AGRADECIMENTOS}

Aos pesquisadores, aos técnicos, aos observadores meteorológicos, aos estagiários e às demais pessoas que de alguma forma contribuíram para a realização deste trabalho; ao
Conselho Nacional de Desenvolvimento Científico e Tecnológico (CNPq), pela concessão de bolsa de produtividade em pesquisa para Alberto Cargnelutti Filho.

\section{REFERÊNCIAS}

BARBETTA, P.A. et al. Estatística para cursos de engenharia e informática. São Paulo: Atlas, 2004. 410p.

BUSSAB, W.O.; MORETTIN, P.A. Estatística básica. 5.ed. São Paulo: Saraiva, 2004. 526p.

CAMPOS, H. de Estatística experimental não-paramétrica. 4.ed. Piracicaba: Departamento de Matemática e Estatística ESALQ, 1983. 349p.

SIEGEL, S.; CASTELLAN JÚNIOR, N.J. Estatística nãoparamétrica para ciências do comportamento. 2.ed. Porto Alegre: Artmed, 2006. 448p.

CARGNELUTTI FILHO, A. et al. Tamanho de amostra para a estimativa das médias decendiais de radiação solar global no estado do Rio Grande do Sul. Ciência e Agrotecnologia, v.31, p.1402-1410, 2007. Disponível em: http://www.scielo.br/ s c i e lo.php? script=s ci_arttext\&pid=S 1413 70542007000500020\&lng=pt\&nrm=iso $>$ Acesso em: 25 jun. 2009. doi: $10.1590 / \mathrm{S} 1413-70542007000500020$.

CARGNELUTTI FILHO, A. et al. Variabilidade temporal e espacial do tamanho de amostra da temperatura mínima do ar no Rio Grande do Sul, Brasil. Ciência Rural, v.36, p.1156-1163, 2006a. Disponível em: http://www.scielo.br/scielo.php?script=sci arttext\&pid=S010384782006000400018\&lng=pt\&nrm=iso $>$ Acesso em: 25 jun. 2009. doi: $10.1590 / \mathrm{S} 0103-84782006000400018$.

CARGNELUTTI FILHO, A. et al. Variabilidade temporal e espacial do tamanho de amostra para estimativa das médias mensais de temperatura máxima do ar no Estado do Rio Grande do Sul. Revista Brasileira de Agrometeorologia, v.14, p.87-95, 2006b.

CARGNELUTTI FILHO, A. et al. Variabilidade temporal e espacial da precisão das estimativas de elementos meteorológicos no Rio Grande do Sul. Ciência Rural, v.39, p.962-970, 2009. Disponível em: http://www.scielo.br/scielo.php?script=sci arttext\&pid=S0103$84782009000400002 \& \operatorname{lng}=$ pt\&nrm=iso $>$ Acesso em: 25 jun. 2009. doi: $10.1590 / \mathrm{S} 0103-84782009005000051$.

FONSECA, J.S.; MARTINS, G.A. Curso de estatística. 5.ed. São Paulo: Atlas, 1995. 317p.

MARTIN, T.N. et al. Regiões homogêneas e tamanho de amostra para atributos do clima no Estado de São Paulo, Brasil. Ciência Rural, v. 38, p.690-697, 2008. Disponível em: http:/ /www.scielo.br/scielo.php? script $=$ sci_arttext\&pid $=$ S0103$84782008000300015 \& \operatorname{lng}=$ pt\&nrm $=$ iso $>$. Acesso em: 25 jun. 2009. doi: $10.1590 / \mathrm{S} 0103-84782008000300015$.

SPIEGEL, R.A. et al. Probabilidade e estatística. 2.ed. Porto Alegre: Bookman, 2004. 398p.

STEEL, R.G.D. et al. Principles and procedures of statistics a biometrical approach. 3.ed. Nova York: McGraw-Hill, 1997. 666p. 